Research Article

\title{
Study on the Evaluation Method of Subgrade Slope Green Protection Effect in Dry-Hot Valley of Sichuan-Tibet Railway
}

\author{
Xueying Bao $(D)$ and Haiwen Li \\ College of Civil Engineering, Lanzhou Jiaotong University, Lanzhou, Gansu 730070, China \\ Correspondence should be addressed to Xueying Bao; baoxueying@mail.lzjtu.cn
}

Received 3 February 2020; Revised 7 May 2020; Accepted 25 May 2020; Published 27 June 2020

Academic Editor: Vincenzo Vespri

Copyright (c) 2020 Xueying Bao and Haiwen Li. This is an open access article distributed under the Creative Commons Attribution License, which permits unrestricted use, distribution, and reproduction in any medium, provided the original work is properly cited.

\begin{abstract}
In view of the harsh environment in the dry-hot valley of Sichuan-Tibet railway and the important role of the green protection of the subgrade slope in railway construction and ecological restoration, this paper evaluates the effect of the green protection of the subgrade slope in dry-hot valleys of the Sichuan-Tibet railway. First, an evaluation index system for the green protection effect of railway subgrade slopes is established in terms of the soil matrix quality, vegetation community quality, and protection performance of the slopes. Second, game theory is adopted to combine the improved-group-analytic-hierarchy-process method and the vector-angle-cosine method and thus determine the weight of each evaluation index. Moreover, the membership-cloudgravity-center method is used to evaluate the green protection level of the railway subgrade slope, and an evaluation cloud map is drawn with the help of MATLAB software to further analyze the evaluation. Finally, a section subgrade slope of the DHV area of the Sichuan-Tibet railway is selected as the evaluation object, and the green protection effect is evaluated to verify the applicability and effectiveness of the model. The study provides a theoretical basis for the protection of subgrade slopes and ecological restoration in the DHV area.
\end{abstract}

\section{Introduction}

With the rapid development of social economy, the large number of exposed slopes caused by the increasing railway construction not only destroyed the vegetation along the railway but also caused a series of ecological problems and geological disasters [1], such as the sudden loss of slope plant species, slope structure damage, landslides, and debris flow [2]. The subgrade slope, as the foundation to maintain the stability of the railway subgrade, is exposed to the air for a long time, so it is strongly influenced by the natural factors, resulting in the strength attenuation; meanwhile, the rock and soil on the slope surface are ruptured, which can easily aggravate the collapse of the slope and cause the subgrade instability [3]. Geological disasters, such as subgrade collapse, landslides, and spalling of the slope surface, may seriously damage the railway and surrounding environment if the subgrade slope is not properly protected. The green protection (plant protection) of a slope not only prevents these disasters but also plays a role in consolidating the slope, growing vegetation along the subgrade slope, and protecting the ecological environment. The green protection of subgrade slopes has therefore become an indispensable part of railway construction. The implementation of ecological protection measures that take into account the engineering effect and landscape function on the subgrade slope, restore the vegetation on the slope surface, prevent the subgrade from destabilizing, and maintain the health of the slope ecosystem has become a hot issue shared by the majority of soil and water conservation and ecological engineering researchers [4].

In recent years, many experts have analyzed the causes of slope instability [5] and destruction [6] through extensive research and found that the main causes of slope damage are mostly rainfall [7], floods [8], and wind action [9] that eroded the soil of the slope, cracked the subgrade slope, reduced the resistance of the subgrade slope to external forces, and eventually caused the slope to collapse and lose 
stability. Against the various causes of subgrade slope instability and destruction, many slope protection technologies and methods have been proposed after a large number of engineering tests, which are basically divided into engineering protection technology and plant protection technology. The forms of railway subgrade slope protection [10] include plant protection of hollow brick-foreign soil, water interception framework protection, hole-window protection wall, assembled framework protection [11], anchor frame beam protection, prestressing force anchor cable antislide pile supporting technology [12], three-dimensional earthwork mesh cushion protection [13], ecological bags, revegetation bags, original ecological tridimensional slope vegetation technology [14], plant fiber blanket road slope protection technology [15], etc. The engineering protection technology is gradually replaced by vegetation slope protection technology because of its high cost, and the protection effect is not as good as vegetation slope protection technology. Vegetation slope protection technology not only saves cost but also green environment and achieves the purpose of slope protection, which is the future development trend.

However, few studies have used mathematical models to evaluate the effect of subgrade slope protection. Moreover, there is no research on the protection of subgrade slopes and related aspects in special areas and special environments, such as the dry-hot valley (DHV) areas. Against the above background, the present paper establishes an evaluation index system for the green protection effect of railway subgrade slopes in a DHV area. Game theory (GT) is then adopted to combine the improved-group-analytic-hierarchy-process (IGAHP) method and the vector-angle-cosine method and thus determine the evaluation index weights. Moreover, a membership-cloud-gravity-center (MCGC) mathematical model is proposed to evaluate the green protection effect of a subgrade slope protection project in a DHV area of the Sichuan-Tibet railway. Since the environment of the DHV area of Sichuan-Tibet railway is very fragile, the environmental protection [16] and ecological restoration of the area are the key issues in the construction of the railway. The study not only improves the lack of evaluation of the subgrade slope protection effect but also provides a theoretical basis for the subgrade slope protection and subsequent monitoring and management of the Sichuan-Tibet railway under construction. Moreover, it provides a quantitative evaluation method for the evaluation of subgrade slope protection and also has a certain reference value for slope protection, environmental protection, and ecological restoration in DHV areas.

\section{Formation and Distribution of DHV Areas}

2.1. Reasons for the Formation of DHV Areas. The DHV areas [17] are formed by the superposition of the original DHV areas and the secondary DHV areas. The original DHV areas already existed, and a special climate was formed due to the combination of special landforms and local microclimates. The area has a tropical and subtropical monsoon climate with a distinct dry and wet season. Moreover, the area has a unique gully system [18], where the foehn effect [19] and local circulation effect of valley wind often occur, causing warming and water loss. Forest vegetation in DHV areas is very difficult to recover. The main reason is that the lack of water makes large areas become barren, especially the loss of surface soil on the slopes of river valleys, which exposes large areas of bare soil and makes the afforestation survival rate low. Secondary DHV areas are formed by the continuous negative disturbance of deforestation. Some studies believe that most of the original vegetation along the banks of the Jinsha River between 3,000 and 5,000 years ago was green broad-leaved oak forests. The above factors have combined to create the extremely fragile ecological environment of the DHV areas. The geomorphological characteristics of DHV areas are shown in Figure 1.

2.2. Distribution and Characteristics of DHV Areas. The DHV areas in China are mainly distributed [17] on the slopes of the valleys of the Minjiang River, Dadu River, Yalong River, Jinsha River, Nujiang River, Lancang River, and other rivers in the southwest of China. The areas are mainly located in the arid and semiarid river-valley areas with latitude of $23^{\circ} 00^{\prime} \sim 28^{\circ} 10^{\prime}$ north, longitude of $98^{\circ}$ $50^{\prime} \sim 103^{\circ} 50^{\prime}$ east, and altitude below $1500 \mathrm{~m}$ [20].

The DHV areas [21] are located in a high-temperature valley area, which is rich in solar and thermal energy resources. It has a hot climate with little rain, severe soil erosion, and fragile ecology. Cold, drought, high wind, fire, and other natural disasters are particularly prominent. The dry season in the DHV areas lasts for more than 8 months each year. The average annual rainfall is about $800 \mathrm{~mm}$ (rainfall is $550 \sim 650 \mathrm{~mm}$, and the dry season rainfall is $50 \sim 160 \mathrm{~mm}$ ). However, the evaporation is more than 3 times the rainfall, the relative humidity is small, and the drought index is about 1.5 to 2.5. The highest surface temperature of bare land reaches $75^{\circ} \mathrm{C}$, and the water content of topsoil within $40 \mathrm{~cm}$ is close to zero, in April and May. Most of the sites in the DHV areas have thick soil, but the vegetation cover is less than 5\%. The vegetation in the DHV areas is seminatural savanna, which has simple structure, low species richness, obvious dominance of herb layer, suboptimal shrub layer, and few trees [17].

\section{Evaluation Index System}

3.1. Analysis of Evaluation Factors. Slope protection engineering involves multidisciplinary and comprehensive system engineering. Therefore, the types and indexes of evaluations of engineering effects must have different properties and characteristics, and problems should be explained at different levels. Meanwhile, such indexes should be interconnected to reflect multiple aspects of the purpose of protection engineering effects comprehensively. From the perspective of system engineering, subgrade slope protection engineering can be regarded as a slope-matrix-vegetation system $[22,23]$. This system and the environment form a unified entity having a certain structure and performance through interaction, intertwining, and infiltration. The construction of a scientifically feasible evaluation 

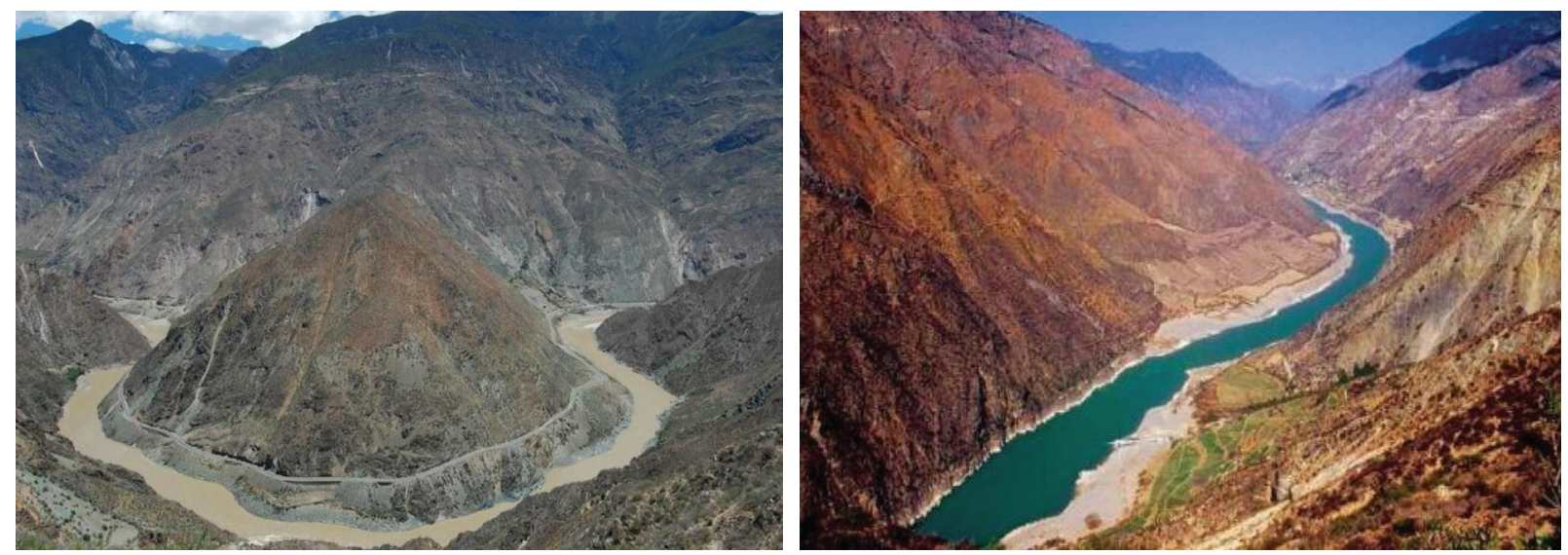

FIGURE 1: Geography of the DHV area.

index system of the engineering effect must therefore be based on the structural integrity and stability and thus the durability of the engineering system. There are two basic units, namely, the matrix and vegetation, of the structure of the slope protection engineering system. The matrix refers to the basic material (i.e., the soil in the present case) provided by the subgrade slope to meet the normal growth of vegetation, which not only provides a reasonable physical structure for vegetation growth but also serves as a platform and carrier [24] for the transformation and utilization of vegetation moisture and nutrients. Meanwhile, the vegetation refers to the plant community on the slope, which is the main biological factor for systematic development and change. The performance of a slope protection engineering system mainly refers to the protection performance of the subgrade slope, which reflects the ability of the slope to resist external environmental interference. According to the structure and performance of the slope-matrix-vegetation system, the soil matrix quality, vegetation community quality, and protection performance are taken as first-level indexes, and second-level evaluation indexes are further analyzed and screened.

The soil in the DHV area is dominated by torrid red soil [25]. This type of soil has a low moisture content, high nutrient loss, and a barren soil layer. Meanwhile, the soil guarantees vegetation survival on the slope in the green protection of the slope. The quality of the soil matrix is therefore a factor that cannot be ignored. The soil matrix quality can be measured by the volumetric weight of soil [26], total soil porosity, soil infiltration rate, soil organic matter and mineral nutrient content, soil expansion and contraction, duration of the soil permanent wilting point (PWP), and the ability of the soil to preserve moisture and fertility [27]. Specifically, the volume weight of soil reflects the comprehensive state of soil organic matter, texture, and compactness; therefore, the volume weight of soil is an important physical characteristic that reflects the quality of the soil matrix. Total soil porosity [25] includes capillary porosity and noncapillary porosity. Capillary porosity refers to the main pore size for soil water retention, and it provides water for plant growth. Noncapillary porosity refers to the main breathable pore size of the soil, and it provides oxygen and nutrients to plant roots in the soil. Moreover, the porosity of the soil affects the infiltration rate [28] of the soil.
The infiltration rate affects the circulation, maintenance, and storage of water in the soil. The water infiltrated into the soil is the main source of water for the plant root system. Torrid red soil is compact and the infiltration rate decreases with increasing depth of the soil layer. The infiltration rate is therefore an important factor in evaluating the quality of the soil matrix. Carbonaceous compounds in soil are the main components of soil organic matter [29] and are one of the main sources of nutrients needed for the growth of slope protection vegetation. The other source is mainly mineral nutrients [30] containing trace elements such as total $\mathrm{N}$, total $\mathrm{P}$, available $\mathrm{P}$, and available $\mathrm{K}$ [31] in the soil. The content of organic matter and mineral nutrients in the soil is therefore an indispensable factor in measuring the quality of the soil matrix. There are distinct dry and wet seasons in the DHV area, resulting in the large expansion and contraction of soil [32] due to temperature changes. The soil expansion and contraction creates cracks in the slope. In the long run, such cracks cause the slope to collapse, and expansion and contraction damages plant root systems and causes plants to die. Therefore, the root systems of slope plants must have a certain tensile strength. Moreover, the alternation of a dry and wet environment affects the mineralization rate of organic carbon in soil and thus the nutrient content of the soil. The soil PWP [33] in the DHV area lasts for a long time, usually 7-8 months, and this is the main cause for the sparse vegetation. Slope protection must therefore improve the quality of the soil matrix and thus shorten the duration of the soil PWP to increase the survival rate of plants. Owing to severe soil coarsening in the DHV area, the soil's ability to retain water and fertilizer is reduced, and soil fertility is reduced. This is a major cause of soil infertility, and the soil coarsening resistance and water and fertilizer retention should thus be improved in slope protection.

The quality of a vegetation community is mainly measured by the vegetation coverage, the vegetation survival rate, incidence of vegetation diseases and insect pests, vegetation species richness index, rationality of vegetation spatial collocation, ability of vegetation to resist drought and heat, and tolerance of vegetation to barrenness. Owing to the hot climate in the DHV area, the soil is relatively infertile and lacks nutrients, resulting in extremely scarce vegetation [34] in this 
area. However, the construction of the railway greatly affected the environment of the area, and plants are therefore planted in the subgrade slope protection project not only to stabilize the subgrade but also to improve the local ecological environment. The numbers and types of slope protection vegetation must meet the requirements of slope protection and environmental improvement in the area, and vegetation coverage [35] can be used as a measure of the number of plants and the area covered by vegetation. Owing to the harsh environment, there are many plant diseases and insect pests in the DHV area, which is a major reason for the low rate of plant survival. The survival rate of plants must therefore be improved by improving the soil and selecting suitable plant species. For slope protection vegetation, shrubs and herbaceous plants that are rich in variety and suitable for DHV growth should be selected. This vegetation has developed roots and strong root meristem, which can further improve the protection ability of slope by improving the soil shear strength. Moreover, vegetation with a strong meristematic ability can improve the coverage of slope vegetation in the short term and enhance the ability of slope stabilization, and vegetation suitable for the area can improve the soil [36] and enhance soil fertility [37]. When planting vegetation, it is also necessary to consider the rationality of the spatial arrangement and distribution. A reasonable structural arrangement can further improve the survival rate of plants and play a positive role in reinforcing the slope. Moreover, the climate of the DHV area is arid and hot; therefore, the selected vegetation should flourish in such a climate.

The protective performance of a slope mainly refers to the slope's resistance to leaching erosion, antiscouring ability, ability to protect against wind and sand, ability to prevent water and soil loss, and ability to prevent natural disasters. Rainfall is concentrated in the DHV area, but evaporation is generally 3-6 times the rainfall [25]. The amount of water infiltrating the soil is too large in the season of concentrated rainfall, and the infiltration water dissolves and leaches nutrients in the soil, such that the slope resistance to leaching erosion is important. Moreover, a large amount of rainwater will scour the slope surface and base, resulting in water and soil loss. Therefore, the slope must also have a strong ability to prevent water and soil loss and scouring. The land is gradually desertified in the DHV area owing to the coarsening of the soil. In addition, there is often windy weather, which causes severe weather such as sandstorms. Therefore, the ability of a slope to resist wind and sand [38] is also important. Moreover, earthquakes, mudslides, landslides, and floods often occur in the area. Subgrade slope protection projects must therefore be able to resist sudden natural disasters and prevent slope collapse from causing serious damage to the environment and railways.

From the above analysis, the more important factors in evaluating the green protection effect of a slope in the DHV area are summarized in Table 1.

3.2. Construction of an Evaluation Index System. Through the above analysis of the soil, vegetation, hydrology, climate, topography, landform, and other geographical features of the DHV area in southwest China and referring to Interim Provisions on Green Protection Techniques for Railway Subgrade Slopes (2003) and relevant research results [39], the three first-level indexes of the soil matrix quality, vegetation community quality, and protective performance of the slope are determined, and an evaluation index system is established through the further refinement and screening of 20 evaluation indexes as second-level indexes, as shown in Figure 2.

A five-level index standard, as described in Table 2, is established on the basis of the evaluation index system, the grading data of organic matter content of a soil survey conducted in China, relevant research results [40-42], and consultation with experts.

\section{Combination Weighting Model}

Methods of determining weights can be divided into subjective weighting methods and objective weighting methods. The disadvantage of a subjective weighting method is the excessive reliance on the opinions of experts while the disadvantage of an objective weighting method is the excessive reliance on quantitative data, but these two methods are complementary. Therefore, to ensure that the weight distribution reflects both the subjective information and objective information, this paper uses GT to combine the weights obtained from both the subjective weighting method (i.e., the IGAHP method) and the objective weighting method (i.e., the vector-angle-cosine method), which make the weighting more reasonable and scientific.

4.1. IGAHP Method. The IGAHP method [43] is based on the traditional analytic hierarchy process (AHP) [44] method and considers the difference between different expert evaluations and assigns a weighting coefficient to each expert using the Euclidean distance to minimize the expert's evaluation bias so that the weight of the index is more reasonable.

The weights of $m$ indexes calculated by the th expert using the AHP method are denoted as

$$
Y^{(t)}=\left(y_{1}^{(t)}, y_{2}^{(t)}, \ldots, y_{m}^{(t)}\right),
$$

where $y_{i}^{(t)}$ is the AHP weight of the $i$ th index given by the $t$ th expert.

The difference between the evaluations of the $g$ th and $h$ th experts is then expressed by the Euclidean distance $d_{g h}(g, h=1,2,3, \ldots, T)$ :

$$
d_{g h}=d\left(Y^{(g)}, Y^{(h)}\right)=\left[\sum_{i-1}^{n}\left(y_{i}^{(g)}-y_{i}^{(h)}\right)^{2}\right]^{(1 / 2)},
$$

where $y_{i}^{(g)}$ and $y_{i}^{(h)}$ are, respectively, the weights of the $i$ th index given by the $g$ th and $h$ th experts, satisfying $d_{t t}=0$ and $d_{g h}=d_{h g} \geq 0$.

Equation (2) shows that closer values of $Y^{(g)}$ and $Y^{(h)}$ (i.e., smaller differences between the evaluations of the two experts) result in smaller $d_{g h}$ if $d_{g h}=0$ and $g \neq h$, then the evaluations of the two experts are exactly the same. 
TABLE 1: Evaluation indexes and description.

\begin{tabular}{|c|c|c|}
\hline First-level indexes & Second-level indexes & Index description \\
\hline \multirow{8}{*}{ Soil matrix quality, $C_{1}$} & $\begin{array}{l}\text { Volumetric weight of soil, } C_{11} \\
\qquad\left(\mathrm{~g} \mathrm{~cm}^{-3}\right)\end{array}$ & $\begin{array}{l}\text { Refers to the mass of the soil per unit volume, reflecting the compactness of } \\
\text { the soil }\end{array}$ \\
\hline & Total soil porosity, $C_{12}(\%)$ & $\begin{array}{l}\text { Includes capillary porosity and noncapillary porosity, reflecting the soil's } \\
\text { ability to breathe and permeate }\end{array}$ \\
\hline & $\begin{array}{l}\text { Organic matter content, } C_{13} \\
\qquad\left(\mathrm{~g} \mathrm{~kg}^{-1}\right)\end{array}$ & $\begin{array}{c}\text { Refers to the amount of all carbon-containing organic compounds present } \\
\text { in the soil }\end{array}$ \\
\hline & $\begin{array}{l}\text { Duration of soil PWP, } C_{14} \\
\text { (month) }\end{array}$ & $\begin{array}{l}\text { Refers to the time that the soil moisture is reduced to the humidity of the soil } \\
\text { when the plant begins to wither }\end{array}$ \\
\hline & Soil infiltration rate, $C_{15}\left(\mathrm{~mm} \mathrm{~h}^{-1}\right)$ & Refers to the amount of infiltration water per unit area of soil per unit time \\
\hline & $\begin{array}{l}\text { Content of mineral nutrients, } C_{16} \\
\qquad\left(\mathrm{~g} \mathrm{~kg}^{-1}\right)\end{array}$ & $\begin{array}{c}\text { Refers to the content of various trace elements, such as total } \mathrm{N} \text {, available } \mathrm{P} \text {, } \\
\text { and available } \mathrm{K} \text {, in the soil }\end{array}$ \\
\hline & $\begin{array}{c}\text { Ability of soil to preserve moisture } \\
\text { and fertility, } C_{17}\end{array}$ & Reflects the soil's abi \\
\hline & $\begin{array}{l}\text { Soil expansion and contraction, } \\
\qquad C_{18}\end{array}$ & $\begin{array}{l}\text { Refers to the ability of the soil to expand and contract through water } \\
\text { absorption and dehydration }\end{array}$ \\
\hline \multirow{7}{*}{$\begin{array}{l}\text { Vegetation community } \\
\text { quality, } C_{2}\end{array}$} & Coverage of vegetation, $C_{21}(\%)$ & $\begin{array}{c}\text { Ratio of the vertical projection area of vegetation (including leaves, stems, } \\
\text { and branches) to the ground }\end{array}$ \\
\hline & Vegetation survival rate, $C_{22}(\%)$ & $\begin{array}{l}\text { Represents the number of plants that survived as a percentage of the total } \\
\text { planted }\end{array}$ \\
\hline & $\begin{array}{c}\text { Incidence of pests and diseases in } \\
\text { vegetation, } C_{23}(\%)\end{array}$ & Refers to the percentage of vegetation with pests and diseases \\
\hline & $\begin{array}{l}\text { Vegetation species richness index, } \\
\qquad C_{24}\end{array}$ & $\begin{array}{c}\text { Refers to the number of species in the community, reflecting the richness of } \\
\text { vegetation species }\end{array}$ \\
\hline & $\begin{array}{l}\text { Rationality of vegetation spatial } \\
\text { collocation, } C_{25}(\%)\end{array}$ & $\begin{array}{l}\text { Reflects the biological characteristics of the vegetation population and the } \\
\text { reasonable degree of intraspecies and interspecies coordination }\end{array}$ \\
\hline & $\begin{array}{l}\text { Ability of vegetation to resist } \\
\text { drought and heat, } C_{26}\end{array}$ & $\begin{array}{c}\begin{array}{c}\text { Reflects the ability of vegetation to resist low temperatures, heat, and } \\
\text { drought }\end{array} \\
\text {. }\end{array}$ \\
\hline & $\begin{array}{l}\text { Vegetation tolerance to } \\
\text { barrenness, } C_{27}\end{array}$ & $\begin{array}{c}\text { Reflects the ability of vegetation to grow in soils that lack nutrients and } \\
\text { water }\end{array}$ \\
\hline \multirow{5}{*}{$\begin{array}{l}\text { Protective performance } \\
\text { of slope, } C_{3}\end{array}$} & $\begin{array}{l}\text { Slope resistance to leaching } \\
\text { erosion, } C_{31}\end{array}$ & $\begin{array}{l}\text { Refers to the ability of the soil matrix to resist the infiltration of water and } \\
\text { leaching of soil nutrients }\end{array}$ \\
\hline & Antiscouring ability, $C_{32}$ & $\begin{array}{c}\text { Refers to the ability of the slope as a whole to resist mechanical damage and } \\
\text { movement such as that of rain and runoff }\end{array}$ \\
\hline & $\begin{array}{l}\text { Ability of slope to protect against } \\
\text { wind and sand, } C_{33}\end{array}$ & $\begin{array}{c}\text { Refers to the ability of slopes to maintain soil and water and prevent severe } \\
\text { weather such as sandstorms }\end{array}$ \\
\hline & $\begin{array}{c}\text { Slope resistance to natural } \\
\text { disasters, } C_{34}\end{array}$ & $\begin{array}{c}\text { Refers to the ability of the slope to resist natural disasters, such as mudslides, } \\
\text { earthquakes, landslides, floods, and collapses }\end{array}$ \\
\hline & $\begin{array}{l}\text { Ability of slope to prevent water } \\
\text { and soil loss, } C_{35}\end{array}$ & $\begin{array}{l}\text { Refers to the ability of the slope to resist damage to soil and land } \\
\text { productivity under external forces }\end{array}$ \\
\hline
\end{tabular}

The sum $d_{t}$ of the values of the degree of similarity of the evaluations made by the $t$ th expert and other experts is expressed by

$$
d_{t}=\sum_{j=1}^{T} d_{t j}, \quad j=1,2, \ldots, T,
$$

where $T$ is the number of experts while $d_{t j}$ is the degree of similarity of the evaluations of the $t$ th and $j$ th experts.

The evaluation weight coefficient $\lambda_{t}$ of the $t$ th expert is calculated as

$$
\begin{cases}\lambda_{1}=\lambda_{2}=\cdots=\lambda_{T}=\frac{1}{T}, & d_{t}=0 \\ \lambda_{t}=\frac{1 / d_{t}}{\sum_{j=1}^{T}\left(1 / d_{j}\right)}, & d_{t} \neq 0 .\end{cases}
$$

In summary, the subjective weight $W_{1}$ determined using the IGAHP method can be calculated as

$$
W_{1}=\sum_{t=1}^{T} \lambda_{t} Y^{(t)}=\sum_{t=1}^{T} \lambda_{t}\left(y_{1}^{(t)}, y_{2}^{(t)}, \ldots, y_{m}^{(t)}\right) \text {. }
$$

\subsection{Vector-Angle-Cosine Method [45]}

Step 1. Construct the optimal value vector $S^{*}$ and worst value vector $s^{*}$ of the evaluation index as

$$
\begin{aligned}
S^{*} & =\left(S_{1}^{*}, S_{2}^{*}, \ldots, S_{m}^{*}\right), \\
s^{*} & =\left(s_{1}^{*}, s_{2}^{*}, \ldots, s_{m}^{*}\right),
\end{aligned}
$$

where $S_{i}^{*}$ and $s_{i}^{*}$ are 


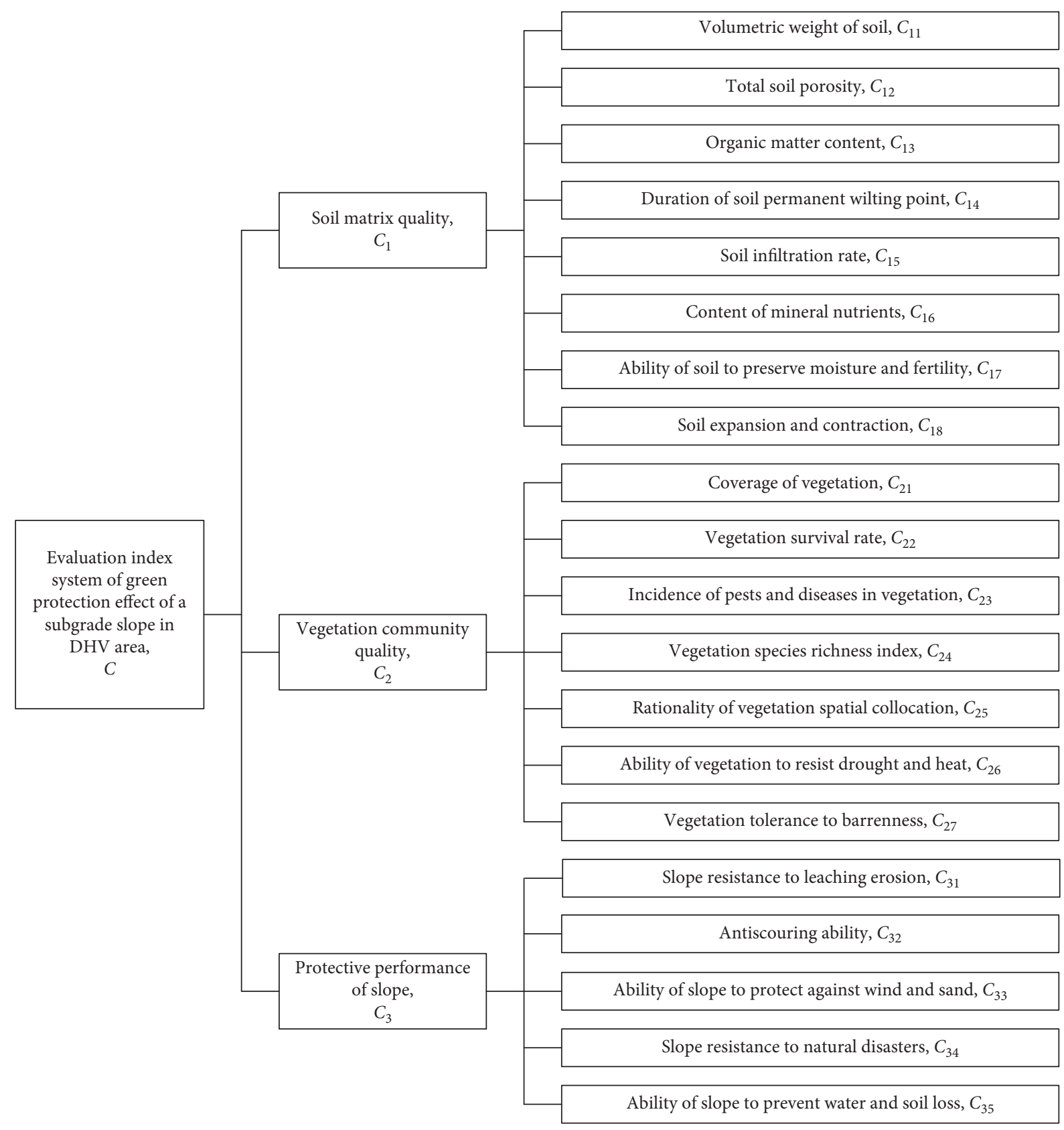

Figure 2: Evaluation index system.

$$
\begin{gathered}
S_{i}^{*}= \begin{cases}\max _{1 \leq j \leq n}\left\{V_{i j}\right\}, & i \in I_{1}, \\
\min _{1 \leq j \leq n}\left\{V_{i j}\right\}, & i \in I_{2},\end{cases} \\
s_{i}^{*}= \begin{cases}\min _{1 \leq j \leq n}\left\{V_{i j}\right\}, & i \in I_{1}, \\
\max _{1 \leq j \leq n}\left\{V_{i j}\right\}, & i \in I_{2},\end{cases}
\end{gathered}
$$

where $S^{*}$ is the optimal value vector of the evaluation index, $S_{i}^{*}$ is the optimal value of the $i$ th evaluation index, $s^{*}$ is the worst evaluation vector of the evaluation index, $s_{i}^{*}$ is the worst evaluation value of the $i$ th evaluation index, $m$ is the number of evaluation indexes, $n$ is the number of experts, $I_{1}$ is a positive index set, $I_{2}$ is a negative index set, and $V_{i j}$ is the basic data of the $i$ th index measured by the $j$ th expert.

Step 2. Calculate the matrix $R$ of the relative deviation between the evaluation object and the optimal value vector and calculate the matrix $\Delta$ of the relative deviation between the evaluation object and the worst-value vector: 
TABLE 2: Indexes' standard level.

\begin{tabular}{|c|c|c|c|c|c|}
\hline Indexes & Excellent & Good & General & Poor & Very poor \\
\hline Volumetric weight of soil $\left(\mathrm{g} \mathrm{cm}^{-3}\right)$ & $<1.1$ & $1.1 \sim 1.2$ & $1.2 \sim 1.3$ & $1.3 \sim 1.4$ & $>1.4$ \\
\hline Total soil porosity (\%) & $>60$ & $60 \sim 56$ & $56 \sim 52$ & $52 \sim 50$ & $<50$ \\
\hline Organic matter content $\left(\mathrm{g} \mathrm{kg}^{-1}\right)$ & $>23.2$ & $17.4 \sim 23.2$ & $11.6 \sim 17.4$ & $3.5 \sim 11.6$ & $<3.5$ \\
\hline Duration of soil PWP (month) & $<2$ & $2 \sim 5$ & $5 \sim 7$ & 7 9 & $>9$ \\
\hline Soil infiltration rate $\left(\mathrm{mm} \mathrm{h}^{-1}\right)$ & $>30$ & $20 \sim 30$ & $10 \sim 20$ & $5 \sim 10$ & $<5$ \\
\hline Total N $\left(\mathrm{g} \cdot \mathrm{kg}^{-1}\right)$ & $>2.0$ & $1.5 \sim 2.0$ & $1 . .0 \sim 1.5$ & $0.75 \sim 1.0$ & $<0.75$ \\
\hline Content of mineral Available $\mathrm{P}\left(\mathrm{P}_{2} \mathrm{O}_{5}\right)\left(\mathrm{g} \mathrm{kg}^{-1}\right)$ & $>0.015$ & $0.01 \sim 0.015$ & $0.005 \sim 0.01$ & $0.002 \sim 0.005$ & $<0.002$ \\
\hline nutrients $\left(\mathrm{g} \mathrm{kg}^{-1}\right) \quad$ Available $\mathrm{K}\left(\mathrm{K}_{2} \mathrm{O}\right)\left(\mathrm{g} \mathrm{kg}^{-1}\right)$ & $>0.1$ & $0.08 \sim 0.1$ & $0.06 \sim 0.08$ & $0.04 \sim 0.06$ & $<0.04$ \\
\hline Ability of soil to preserve moisture and fertility & Very strong & Strong & Medium & Weak & Very weak \\
\hline Soil expansion and contraction & Very weak & Weak & Medium & Strong & Very strong \\
\hline Coverage of vegetation $(\%)$ & $>90$ & $80 \sim 90$ & $70 \sim 80$ & $50 \sim 70$ & $<50$ \\
\hline Vegetation survival rate (\%) & $>90$ & $80 \sim 90$ & $70 \sim 80$ & $50 \sim 70$ & $<50$ \\
\hline Incidence of pests and diseases in vegetation (\%) & $<20$ & $20 \sim 40$ & $40 \sim 70$ & $70 \sim 90$ & $>90$ \\
\hline Vegetation species richness index & $>1.0$ & $0.8 \sim 1.0$ & $0.6 \sim 0.8$ & $0.4 \sim 0.6$ & $<0.4$ \\
\hline Rationality of vegetation spatial collocation & $\begin{array}{l}\text { Extremely } \\
\text { reasonable }\end{array}$ & Reasonable & Medium & Unreasonable & Extremely unreasonable \\
\hline Ability of vegetation to resist drought and heat & Very strong & Strong & Medium & Weak & Very weak \\
\hline Vegetation tolerance to barrenness & Very strong & Strong & Medium & Weak & Very weak \\
\hline Slope resistance to leaching erosion & Very strong & Strong & Medium & Weak & Very weak \\
\hline Antiscouring ability & Very strong & Strong & Medium & Weak & Very weak \\
\hline Ability of slope to protect against wind and sand & Very strong & Strong & Medium & Weak & Very weak \\
\hline Slope resistance to natural disasters & Very strong & Strong & Medium & Weak & Very weak \\
\hline Ability of slope to prevent water and soil loss & Very strong & Strong & Medium & Weak & Very weak \\
\hline
\end{tabular}

$$
\begin{aligned}
& R=\left(r_{i j}\right)_{m \times n}=\left(\frac{\left|V_{i j}-S_{i}^{*}\right|}{\max _{1 \leq j \leq n}\left(V_{i j}\right)-\min _{1 \leq j \leq n}\left(V_{i j}\right)}\right)_{m \times n}, \\
& \Delta=\left(\delta_{i j}\right)_{m \times n}=\left(\frac{\left|V_{i j}-s_{i}^{*}\right|}{\max _{1 \leq j \leq n}\left(V_{i j}\right)-\min _{1 \leq j \leq n}\left(V_{i j}\right)}\right)_{m \times n},
\end{aligned}
$$

where $r_{i j}$ is the optimal value of the relative deviation of the $i$ th index calculated from the basic data given by the $j$ th expert while $\delta_{i j}$ is the worst value of the relative deviation of the $i$ th index calculated from the basic data given by the $j$ th expert.

Step 3. Determine the value of the vector angle cosine $c_{i}$ of the evaluation index (equation (12)) and then normalize it to obtain the objective weight $W_{2}$ (equation (13)):

$$
\begin{aligned}
c_{i} & =\cos \left\langle r_{i}, \delta_{i}\right\rangle=\frac{\sum_{j=1}^{n} r_{i j} \delta_{i j}}{\sqrt{\sum_{j=1}^{n} r_{i j}^{2}} \sqrt{\sum_{j=1}^{n} \delta_{i j}^{2}}}, \\
W_{2} & =\left(w_{1}^{C}, w_{2}^{C}, \ldots, w_{m}^{C}\right)^{T},
\end{aligned}
$$

where the equation $w_{i}^{C}=c_{i} / \sum_{i=1}^{m} c_{i}$ is used in normalization and $w_{i}^{C}$ is the vector-angle-cosine weight of the $i$ th index.

4.3. GT Combination Weighting Model. The GT [46, 47] combination weighting model is constructed by finding the minimum deviation between subjective and objective weights to obtain a compromise value considering the conflict between subjective and objective weights. This achieves the effect of making an interactive decision between subjective and objective weights, so as to obtain the optimal combination of weights.

Supposing $\theta_{1}$ and $\theta_{2}$ are the weighting coefficients of subjective and objective weights and $L$ is the number of methods used to determine the weights, the combination weight $W^{*}$ is calculated as

$$
W^{*}=\sum_{k=1}^{L} \theta_{k} \cdot W_{k}^{T}=\theta_{1} W_{1}^{T}+\theta_{2} W_{2}^{T},
$$

where $W_{1}^{T}$ and $W_{2}^{T}$ are the transposed matrices of $W_{1}$ and $W_{2}$.

Specifically, the combination weights are obtained by the following calculation.

The values of $\theta_{1}$ and $\theta_{2}$ are optimized according to the aggregation model of GT:

$$
\left\{\begin{array}{l}
\min \left\|\theta_{1} W_{1}^{T}+\theta_{2} W_{2}^{T}-W_{1}^{T}\right\|_{2}, \\
\min \left\|\theta_{1} W_{1}^{T}+\theta_{2} W_{2}^{T}-W_{2}^{T}\right\|_{2} .
\end{array}\right.
$$

According to the differential characteristics of the matrix, the optimal first derivative is obtained from equation (15):

$$
\left\{\begin{array}{l}
\theta_{1} W_{1} W_{1}^{T}+\theta_{2} W_{1} W_{2}^{T}=W_{1} W_{1}^{T}, \\
\theta_{1} W_{2} W_{1}^{T}+\theta_{2} W_{2} W_{2}^{T}=W_{2} W_{2}^{T} .
\end{array}\right.
$$

The weighted coefficients $\theta_{1}$ and $\theta_{2}$ are obtained by solving equation (16) and then normalizing the solution: 


$$
\begin{aligned}
& \theta_{1}^{*}=\frac{\theta_{1}}{\theta_{1}+\theta_{2}}, \\
& \theta_{2}^{*}=\frac{\theta_{2}}{\theta_{1}+\theta_{2}},
\end{aligned}
$$

where $\theta_{1}^{*}$ and $\theta_{2}^{*}$ are the normalized values of $\theta_{1}$ and $\theta_{2}$.

The optimized combination weight $W^{*}$ is then calculated as

$$
W^{*}=\sum_{k=1}^{L} \theta_{k} \cdot W_{k}^{T}=\theta_{1}^{*} W_{1}^{T}+\theta_{2}^{*} W_{2}^{T} .
$$

\section{Evaluation Model}

5.1. MCGC Model. The MCGC model [48] is a comprehensive evaluation method suitable for solving uncertainty and fuzziness problems. Through the conversion of qualitative concepts and quantitative data, the evaluation and measurement of the uncertainty problem are realized.

Suppose that $U$ is a quantitative domain composed of precise values and $C$ is a qualitative concept on the domain $U$. For any element $x$, there is a random number $\mu(x) \in[0,1]$ with stable tendency, which is called the membership degree of $x$ to the qualitative concept $C$. The distribution of membership degree $\mu$ on the domain $U$ forms the membership cloud $C(x)$, and $[x, \mu(x)]$ constitutes a cloud drop [49]. The expectation Ex, entropy En, and hyperentropy $\mathrm{He}$ are introduced to represent the eigenvalues of the cloud. The expectation Ex is the point value on the domain $U$ that can best represent the fuzzy qualitative concept $C$, indicating the central value of the fuzzy concept $C$ in the domain, which is the central distribution of the cloud. Entropy En is the degree of uncertainty of qualitative concept $C$, which reflects the random probability of cloud droplet of qualitative concept $C$ and the acceptable range of cloud droplet of qualitative concept $C$ in domain $U$, and the greater the entropy value, the greater the randomness. Hyperentropy $\mathrm{He}$ is a measure of the uncertainty of entropy, which reflects the degree of cloud dispersion and cloud thickness, as shown in Figure 3.

In Figure 3, Ex is the average of the cloud, which represents the qualitative concept. En represents the value range of qualitative concepts in the domain space, which reflects the "wide" and "narrow" of the cloud. He represents the randomness of the sample values and provides a method to combine the ambiguity and randomness, which reflects the "thickness" and "thinness" of the cloud [50].

\subsection{Determining the Eigenvalues of the Standard Evaluation} Level. Experts in relevant fields, such as railway subgrade slope construction, slope green protection, and the natural environment of the DHV area, were invited to give numerical intervals for the five evaluation levels of excellent, good, general, poor, and very poor. The inverse cloud generator of MATLAB software was used to generate eigenvalues of the standard evaluation level. The relevant formulas are

$$
\left\{\begin{array}{l}
\operatorname{Ex}_{i j}=\frac{R_{j \min }^{i}+R_{j \max }^{i}}{2}, \\
\operatorname{En}_{i j}=\frac{R_{j \max }^{i}-R_{j \min }^{i}}{6}, \\
\mathrm{He}_{i j}=k,
\end{array}\right.
$$

where $\quad\left(R_{j}^{i}=\left(R_{j \text { min }}^{i}, R_{j \max }^{i}\right), i=1,2, \ldots, n ; j=1,2, \ldots, 5\right)$ is the numerical interval of the $j$ th standard evaluation level given by the $i$ th expert; $\mathrm{Ex}_{i j}, \mathrm{En}_{i j}$, and $\mathrm{He}_{i j}$ are the values of expectation, entropy, and hyperentropy obtained from the numerical intervals of the standard evaluation levels given by each expert; and $k$ is a constant that can be set according to needs.

Assuming that the weights of the evaluation experts are the same, the eigenvalues of the standard evaluation level are

$$
\left\{\begin{array}{l}
\mathrm{Ex}_{j}=\frac{\mathrm{Ex}_{1 j} \mathrm{En}_{1 j}+\mathrm{Ex}_{2 j} \mathrm{En}_{2 j}+\cdots+\mathrm{Ex}_{n j} \mathrm{En}_{n j}}{\mathrm{En}_{1 j}+\mathrm{En}_{2 j}+\cdots+\mathrm{En}_{n j}}, \\
\mathrm{En}_{j}=\mathrm{En}_{1 j}+\mathrm{En}_{2 j}+\cdots+\mathrm{En}_{n j}, \\
\mathrm{He}_{j}=\frac{\mathrm{He}_{1 j} \mathrm{En}_{1 j}+\mathrm{He}_{2 j} \mathrm{En}_{2 j}+\cdots+\mathrm{He}_{n j} \mathrm{En}_{n j}}{\mathrm{En}_{1 j}+\mathrm{En}_{2 j}+\cdots+\mathrm{En}_{n j}},
\end{array}\right.
$$

where $\mathrm{Ex}_{i j}, \mathrm{En}_{i j}$, and $\mathrm{He}_{i j}$ are the eigenvalues obtained by integrating the expectation, entropy, and hyperentropy calculated from the numerical intervals of the $j$ th standard evaluation level given by all experts.

5.3. Aggregation of Evaluation Indexes. Index aggregation refers to determining the corresponding standard evaluation level according to the data of second-level indexes collected by each expert to obtain the eigenvalues of second-level indexes and then performing parallel aggregation to obtain the weighted eigenvalues of second-level indexes. Finally, vertical upward aggregation is performed to obtain the eigenvalues of first-level indexes and the eigenvalues of the evaluation result.

5.3.1. Parallel Aggregation.

$$
\left\{\begin{array}{l}
\mathrm{Ex}=\frac{\sum_{i=1}^{n}\left(\mathrm{Ex}_{i} \times \delta_{i}\right)}{\sum_{t=1}^{n} \delta_{i}}, \\
\mathrm{En}=\frac{\sum_{i=1}^{n}\left(\mathrm{En}_{i} \times \delta_{i}\right)}{\sum_{i=1}^{n} \delta_{i}^{2}}, \\
\mathrm{He}=\frac{\sum_{i=1}^{n}\left(\mathrm{He}_{i} \times \delta_{i}^{2}\right)}{\sum_{i=1}^{n} \delta_{i}^{2}},
\end{array}\right.
$$




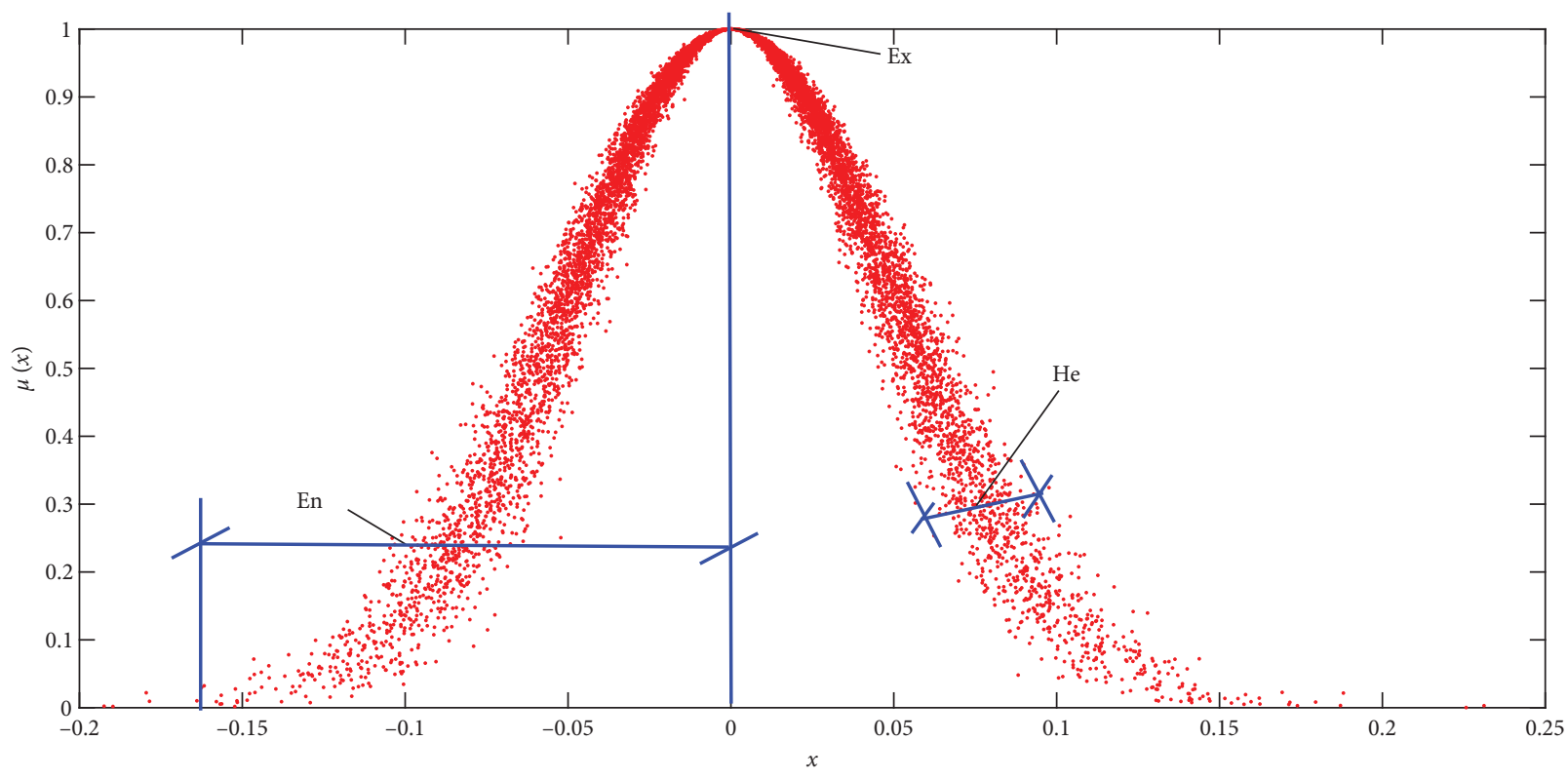

Figure 3: Principle of cloud map.

where $\delta_{i}(i=1,2, \ldots, n)$ denotes the weight of the $i$ th expert (i.e., the expert weight coefficient determined using the IGAHP method) while Ex, En, and He, respectively, refer to the expectation, entropy, and hyperentropy of the secondlevel indexes after parallel aggregation.

\subsection{2.}

Vertical Upward

Aggregation.

$$
\left\{\begin{array}{l}
\mathrm{Ex}=\frac{\sum_{i=1}^{m}\left(\mathrm{Ex}_{i} \times \mathrm{En}_{i} \times W_{i}^{*}\right)}{\sum_{i=1}^{m}\left(\mathrm{En}_{i} \times W_{i}^{*}\right)}, \\
\mathrm{En}=\sum_{i=1}^{m}\left(\mathrm{En}_{i} \times W_{i}^{*}\right), \\
\mathrm{He}=\frac{\sum_{i=1}^{m}\left(\mathrm{He}_{i} \times \mathrm{En}_{i} \times W_{i}^{*}\right)}{\sum_{i=1}^{m}\left(\mathrm{En}_{i} \times W_{i}^{*}\right)},
\end{array}\right.
$$

where $W_{i}^{*}(i=1,2, \ldots, m)$ is the weight of the GT combination of the $i$ th index while Ex, En, and He, respectively, refer to the expectation, entropy, and hyperentropy after vertical upward aggregation.

5.4. Generating a Cloud Map of Evaluation Results. The eigenvalues of the evaluation result are finally obtained through parallel aggregation and vertical upward polymerization. The eigenvalues of the evaluation result and the eigenvalues of the five evaluation levels are input to the forward cloud generator of MATLAB software to obtain the cloud map of evaluation results.

\section{Case Study}

6.1. Generating Eigenvalues of the Standard Evaluation Level.
The standard evaluation levels of excellent, good, general, poor, and very poor do not have precise numerical intervals; i.e., there are no eigenvalues of the standard evaluation level. Therefore, two experts on the construction of railway subgrade slopes, two experts on the green protection of subgrade slopes, and two experts on the natural environment in the DHV area were invited to give numerical intervals for the five standard evaluation levels. The intervals are given in Table 3.

The numerical intervals in Table 3 and equations (19) and (20) are input into the reverse cloud generator of MATLAB software to obtain the eigenvalue of the standard evaluation level, as shown in Table 4.

6.2. Engineering Case. The Sichuan-Tibet railway runs from Chengdu, the capital of Sichuan, to Lhasa, the capital of Xizang, which is divided into three sections: Chengdu to Ya'an (Chengya section), Ya'an to Nyingchi (Yalin section), and Nyingchi to Lhasa (Lalin section). The Chengya section was officially opened to traffic on December 28, 2018, the Lalin section has now entered the track-laying phase, and the most difficult Yalin section is now under construction. Most areas the railway passes through are mountains, hills, and valleys, where the climate is extremely harsh and natural disasters such as floods, sandstorms, and earthquakes are common. In particular, Luding and surrounding areas of the Dadu River Basin, Batang and surrounding areas of the Jinsha River Basin, and parts of the Yapan River and other areas along the Sichuan-Tibet railway have DHV areas.

After consulting the six experts as described above, a section of the railway from Ya'an to Kangding of the Sichuan-Tibet railway, which is mainly located in Dadu River Basin in Luding County and passes through DHV area, is selected as the research object. The area has a subhumid climate on the Qinghai-Tibet Plateau and is influenced by 
TABLE 3: Numerical interval values of standard evaluation levels.

\begin{tabular}{|c|c|c|c|c|c|}
\hline Experts & Excellent & Good & General & Poor & Very poor \\
\hline 1 & $(0.82,0.96)$ & $(0.70,0.81)$ & $(0.60,0.69)$ & $(0.47,0.59)$ & $(0.33,0.46)$ \\
\hline 2 & $(0.84,0.97)$ & $(0.73,0.83)$ & $(0.62,0.72)$ & $(0.50,0.61)$ & $(0.35,0.49)$ \\
\hline 3 & $(0.86,0.98)$ & $(0.74,0.85)$ & $(0.61,0.73)$ & $(0.48,0.60)$ & $(0.33,0.47)$ \\
\hline 4 & $(0.83,0.96)$ & $(0.74,0.82)$ & $(0.59,0.73)$ & $(0.45,0.58)$ & $(0.31,0.44)$ \\
\hline 5 & $(0.82,0.93)$ & $(0.75,0.81)$ & $(0.64,0.74)$ & $(0.51,0.63)$ & $(0.32,0.50)$ \\
\hline 6 & $(0.85,0.95)$ & $(0.73,0.84)$ & $(0.61,0.72)$ & $(0.46,0.60)$ & $(0.32,0.45)$ \\
\hline
\end{tabular}

TABLE 4: Eigenvalue of standard evaluation levels.

\begin{tabular}{lccccc}
\hline Eigenvalue & Excellent & Good & General & Poor & Very poor \\
\hline $\mathrm{Ex}^{0}$ & 0.8976 & 0.7790 & 0.6667 & 0.5392 & 0.3985 \\
$\mathrm{En}^{0}$ & 0.0608 & 0.0475 & 0.0550 & 0.0617 & 0.0708 \\
$\mathrm{He}^{0}$ & 0.0062548 & 0.0063105 & 0.0062621 & 0.0062986 & 0.0062808 \\
\hline
\end{tabular}

the southeast and southwest monsoons and the cold air on the Qinghai-Tibet Plateau. The annual precipitation is $664.4 \mathrm{~mm}$, and the evaporation is $1480.9 \mathrm{~mm}$. November to April of the next year is the dry season, which is a typical DHV area [51]. The soil types mainly include red soil, mountain brown soil, and mostly leached soil, which is very likely to cause geological disasters. Vegetation is mostly alpine shrubs and meadows, as well as Opuntia sp., succulent shrubs, and Acacia farnesiana and Pistacia weinmannifolia communities. The six experts were invited to select different locations of the subgrade slope project to measure the index data through on-site investigation and sample testing. Specifically, the subordinate indexes of soil matrix quality, such as volumetric weight of soil and total soil porosity, are measured by the cutting ring method, and the soil infiltration rate and organic matter content are measured by the round block technique and the CODcr method, respectively. The subordinate indexes of vegetation community quality are determined by the plant community sample survey method, and the species richness index refers to the Shannon-Wiener index. The strength of qualitative indexes such as soil expansion and contraction, antiscouring ability, ability of slope to protect against wind, and sand is given by experts using the visual method according to the actual situation of the study area. The specific data are shown in Table 5.

The evaluation levels of each second-level index are obtained from the basic data in Tables 2-5, as shown in Table 6 .

\subsection{Determining the Combined Weight}

6.3.1. IGAHP Weights. The six experts used the AHP method to analyze and compare the importance of the firstlevel indexes of the soil matrix quality $C_{1}$, vegetation community quality $C_{2}$, and protective performance of the slope $C_{3}$ and then provided their judgment matrix, which passed a consistency test, as shown in Table 7 .

According to the judgment matrix in Table 7, the firstlevel index weights $Y^{(t)}$ determined using the AHP method are obtained, and these weights are then substituted into equations (1)-(4) to obtain the expert's weight coefficient $\lambda$ :

$\lambda=(0.2161,0.1512,0.1792,0.1498,0.0954,0.2083)$.
The weight coefficient $\lambda$ of experts and the first-level index weights $Y^{(t)}$ determined using the AHP method are substituted into equation (5) to obtain the IGAHP weight of the first-level indexes:

$$
W_{1}=(0.3403,0.5396,0.1179) \text {. }
$$

Similarly, the IGAHP weights of second-level indexes are obtained, as shown in Table 8 . The detailed calculation process is not described here.

6.3.2. Vector-Angle-Cosine Weights. For the qualitative indexes of the evaluation index system, supposing a score of 10 is the highest score, experts provided a score for the actual conditions. The weight of the vector-angle-cosine method of the first-level indexes is obtained by substituting the scores of the first-level indexes given by experts into equations (6)-(13):

$$
W_{2}=(0.2864,0.4045,0.3091) \text {. }
$$

Similarly, the data of quantitative indexes in Table 5 and the scores of qualitative second-level indexes given by experts are substituted into equations (6)-(13), and the weights of the vector-angle-cosine method for the second-level indexes are then obtained, as shown in Table 8. The scores of qualitative indexes and the detailed calculation process are not provided here.

6.3.3. Combined Weights. The GT combination coefficients of the first-level indexes are obtained by substituting the subjective and objective weights of the first-level indexes determined by the IGAHP method and the vector-anglecosine method into equations (14)-(17):

$$
\begin{aligned}
& \theta_{1}^{*}=0.4257, \\
& \theta_{2}^{*}=0.5743 .
\end{aligned}
$$

According to equation (18) and the subjective and objective weights calculated above, the combined weights of the first-level indicators are obtained: 
TABle 5: Index data.

\begin{tabular}{|c|c|c|c|c|c|c|}
\hline Indexes & 1 & 2 & 3 & 4 & 5 & 6 \\
\hline Volumetric weight of soil $\left(\mathrm{g} \cdot \mathrm{cm}^{-3}\right)$ & 1.15 & 1.19 & 1.23 & 1.13 & 1.11 & 1.21 \\
\hline Total soil porosity (\%) & 57.4 & 59.8 & 61.3 & 58.2 & 56.7 & 62.5 \\
\hline Organic matter content $\left(\mathrm{g} \cdot \mathrm{kg}^{-1}\right)$ & 18.2 & 17.6 & 17.3 & 19.4 & 18.5 & 17.9 \\
\hline Duration of soil PWP (month) & 4 & 4 & 3 & 3 & 4 & 6 \\
\hline Soil infiltration rate $\left(\mathrm{mm} \cdot \mathrm{h}^{-1}\right)$ & 27.3 & 26.5 & 29.7 & 31.2 & 28.4 & 32.2 \\
\hline Total N $\left(\mathrm{g} \cdot \mathrm{kg}^{-1}\right)$ & 1.49 & 1.8 & 1.5 & 1.4 & 2.05 & 1.8 \\
\hline Available $\mathrm{P}\left(\mathrm{P}_{2} \mathrm{Q}_{5}\right)\left(\mathrm{g} \cdot \mathrm{kg}^{-1}\right)$ & 0.013 & 0.014 & 0.016 & 0.018 & 0.019 & 0.013 \\
\hline Available $\mathrm{K}\left(\mathrm{K}_{2} \mathrm{O}\right)\left(\mathrm{g} \cdot \mathrm{kg}^{-1}\right)$ & 0.09 & 0.095 & 0.093 & 0.088 & 0.11 & 0.07 \\
\hline Ability of soil to preserve moisture and fertility & Strong & Strong & Medium & Strong & Medium & Strong \\
\hline Soil expansion and contraction & Weak & Weak & Weak & Medium & Weak & Weak \\
\hline Coverage of vegetation (\%) & 91.3 & 88.4 & 89.7 & 87.9 & 88.2 & 90.8 \\
\hline Vegetation survival rate $(\%)$ & 81.7 & 89.2 & 84.5 & 91.5 & 79.6 & 86.1 \\
\hline Incidence of pests and diseases in vegetation (\%) & 34.5 & 41.7 & 40.9 & 35.3 & 40.1 & 37.5 \\
\hline Vegetation species richness index & 0.97 & 1.03 & 0.95 & 0.99 & 1.06 & 0.92 \\
\hline Rationality of vegetation spatial collocation & Reasonable & Reasonable & Reasonable & Reasonable & Reasonable & Reasonable \\
\hline Ability of vegetation to resist drought and heat & Very strong & Strong & Strong & Strong & Strong & Strong \\
\hline Vegetation tolerance to barrenness & Strong & Strong & Very strong & Strong & Strong & Strong \\
\hline Slope resistance to leaching erosion & Strong & Strong & Strong & Strong & Very strong & Strong \\
\hline Antiscouring ability & Strong & Strong & Medium & Strong & Medium & Strong \\
\hline Ability of slope to protect against wind and sand & Strong & Medium & Strong & Strong & Strong & Strong \\
\hline Slope resistance to natural disasters & Medium & Strong & Strong & Medium & Strong & Strong \\
\hline Ability of slope to prevent water and soil loss & Strong & Very strong & Strong & Strong & Strong & Medium \\
\hline
\end{tabular}

TAвLE 6: Evaluation level of second-level indexes.

\begin{tabular}{|c|c|c|c|c|c|c|c|}
\hline First-level indexes & Second-level indexes & 1 & 2 & 3 & 4 & 5 & 6 \\
\hline \multirow{8}{*}{$C_{1}$} & $C_{11}$ & Good & Good & General & Good & Good & General \\
\hline & $C_{12}$ & Good & Good & Excellent & Good & Good & Excellent \\
\hline & $C_{13}$ & Good & Good & General & Good & Good & Good \\
\hline & $C_{14}$ & Good & Good & Good & Good & Good & General \\
\hline & $C_{15}$ & Good & Good & Good & Excellent & Good & Excellent \\
\hline & $C_{16}$ & General & Good & Good & Good & Excellent & Good \\
\hline & $C_{17}$ & Good & Good & General & Good & General & Good \\
\hline & $C_{18}$ & Good & Good & Good & General & Good & Good \\
\hline \multirow{7}{*}{$C_{2}$} & $C_{21}$ & Excellent & Good & Good & Good & Good & Excellent \\
\hline & $C_{22}$ & Good & Good & Good & Excellent & General & Excellent \\
\hline & $C_{23}$ & Good & General & General & Good & General & Good \\
\hline & $C_{24}$ & Good & Excellent & Good & Good & Excellent & Good \\
\hline & $C_{25}$ & Good & Good & Good & Good & Good & Good \\
\hline & $C_{26}$ & Excellent & Good & Good & Good & Good & Good \\
\hline & $C_{27}$ & Good & Good & Excellent & Good & Good & Good \\
\hline \multirow{5}{*}{$C_{3}$} & $C_{31}$ & Good & Good & Good & Good & Excellent & Good \\
\hline & $C_{32}$ & Good & Good & General & Good & General & Good \\
\hline & $C_{33}$ & Good & General & Good & Good & Good & Good \\
\hline & $C_{34}$ & General & Good & Good & General & Good & Good \\
\hline & $C_{35}$ & Good & Excellent & Good & Good & Good & General \\
\hline
\end{tabular}

TABLE 7: Judgment matrix for first-level indexes.

\begin{tabular}{ccccccccccccccccccc}
\hline$C$ & \multicolumn{1}{c}{$C_{1}$} & \multicolumn{1}{c}{$C_{2}$} & \multicolumn{1}{c}{$C_{3}$} \\
\hline$C_{1}$ & 1 & 1 & 1 & 1 & 1 & 1 & $1 / 2$ & $1 / 3$ & $1 / 3$ & 1 & 3 & $1 / 2$ & 3 & 2 & 2 & 3 & 4 & 5 \\
$C_{2}$ & 2 & 3 & 3 & 1 & $1 / 3$ & 2 & 1 & 1 & 1 & 1 & 1 & 1 & 4 & 7 & 4 & 2 & 3 & 6 \\
$C_{3}$ & $1 / 3$ & $1 / 2$ & $1 / 2$ & $1 / 3$ & $1 / 4$ & $1 / 5$ & $1 / 4$ & $1 / 7$ & $1 / 4$ & $1 / 2$ & $1 / 3$ & $1 / 6$ & 1 & 1 & 1 & 1 & 1 & 1 \\
\hline
\end{tabular}


TABLE 8: Weights of second-level indexes.

\begin{tabular}{lccc}
\hline Indexes & Weights of the IGAHP method & Weights of the vector-angle-cosine method & GT combination weights \\
\hline$C_{11}$ & 0.0439 & 0.0529 & 0.0487 \\
$C_{12}$ & 0.0418 & 0.0522 & 0.0474 \\
$C_{13}$ & 0.0501 & 0.0646 & 0.0579 \\
$C_{14}$ & 0.0399 & 0.0527 & 0.0468 \\
$C_{15}$ & 0.0352 & 0.0541 & 0.0453 \\
$C_{16}$ & 0.0654 & 0.0570 & 0.0609 \\
$C_{17}$ & 0.0337 & 0.0340 & 0.0339 \\
$C_{18}$ & 0.0612 & 0.0740 & 0.0681 \\
$C_{21}$ & 0.0455 & 0.0405 & 0.0428 \\
$C_{22}$ & 0.0379 & 0.0598 & 0.0496 \\
$C_{23}$ & 0.0401 & 0.0430 & 0.0417 \\
$C_{24}$ & 0.0520 & 0.0625 & 0.0576 \\
$C_{25}$ & 0.0271 & 0.0333 & 0.0304 \\
$C_{26}$ & 0.0466 & 0.0570 & 0.0522 \\
$C_{27}$ & 0.0297 & 0.0364 & 0.0333 \\
$C_{31}$ & 0.0406 & 0.0497 & 0.0455 \\
$C_{32}$ & 0.0263 & 0.0292 & 0.0279 \\
$C_{33}$ & 0.0491 & 0.0659 & 0.0581 \\
$C_{34}$ & 0.0262 & 0.0315 & 0.0291 \\
$C_{35}$ & 0.0394 & 0.0497 & 0.0449 \\
\hline
\end{tabular}

TABLE 9: Eigenvalue of second-level indexes after parallel aggregation.

\begin{tabular}{|c|c|c|c|c|}
\hline First-level indexes & Second-level indexes & Ex & En & $\mathrm{He}$ \\
\hline \multirow{8}{*}{$C_{1}$} & $C_{11}$ & 0.735484 & 0.050706 & 0.0062901 \\
\hline & $C_{12}$ & 0.824958 & 0.053186 & 0.0062871 \\
\hline & $C_{13}$ & 0.758876 & 0.048864 & 0.0062937 \\
\hline & $C_{14}$ & 0.755608 & 0.049343 & 0.0062990 \\
\hline & $C_{15}$ & 0.821471 & 0.052458 & 0.0062901 \\
\hline & $C_{16}$ & 0.766046 & 0.050169 & 0.0062795 \\
\hline & $C_{17}$ & 0.748162 & 0.049250 & 0.0062996 \\
\hline & $C_{18}$ & 0.762177 & 0.048453 & 0.0062975 \\
\hline \multirow{7}{*}{$C_{2}$} & $C_{21}$ & 0.829334 & 0.054285 & 0.0062824 \\
\hline & $C_{22}$ & 0.803704 & 0.052844 & 0.0062901 \\
\hline & $C_{23}$ & 0.731183 & 0.050221 & 0.0062983 \\
\hline & $C_{24}$ & 0.808247 & 0.049907 & 0.0063009 \\
\hline & $C_{25}$ & 0.779000 & 0.047500 & 0.0063110 \\
\hline & $C_{26}$ & 0.804629 & 0.051017 & 0.0062962 \\
\hline & $C_{27}$ & 0.800253 & 0.050604 & 0.0063008 \\
\hline \multirow{5}{*}{$C_{3}$} & $C_{31}$ & 0.790314 & 0.048185 & 0.0063081 \\
\hline & $C_{32}$ & 0.748162 & 0.049250 & 0.0062996 \\
\hline & $C_{33}$ & 0.762020 & 0.048471 & 0.0063047 \\
\hline & $C_{34}$ & 0.737909 & 0.050436 & 0.0062918 \\
\hline & $C_{35}$ & 0.773540 & 0.051064 & 0.0062917 \\
\hline
\end{tabular}

$$
W^{*}=(0.3093,0.4620,0.2287)
$$

Similarly, the GT combination coefficient of the second-level indexes is obtained and the combined weights of the second-level indexes are then obtained, as shown in Table 8 . The detailed calculation process is not described here.

6.4. Index Aggregation. According to the calculation of the subjective weight adopting the IGAHP, the weight of each expert is $\delta=\lambda=(0.2161,0.1512,0.1792,0.1498,0.0954$, $0.2083)$.
Substituting the eigenvalues of the second-level indexes obtained according to Tables 4-6 and the weight of the experts into equation (21) gives the eigenvalues of the second-level indexes after parallel aggregation, as shown in Table 9.

According to the values in Tables 8 and 9, vertical upward aggregation is performed using equation (22), and the eigenvalues of the first-level indexes and the evaluation result are obtained, as shown in Table 10.

6.5. Cloud Map of Evaluation Results. The eigenvalues $\left(\mathrm{Ex}^{0}, \mathrm{En}^{0}, \mathrm{He}^{0}\right)$ of the standard evaluation levels in Table 4 
TABLE 10: Eigenvalue of indexes after vertical upward aggregation.

\begin{tabular}{lcrr}
\hline Evaluation result & Eigenvalue & First-level indexes & \multicolumn{1}{c}{ Eigenvalues } \\
\hline & & $C_{1}$ & $(0.775145,0.018342,0.0062919)$ \\
$C$ & $\left(\mathrm{Ex}^{*}, \mathrm{En}^{*}, \mathrm{He}^{*}\right)=(0.781816,0.018719,0.0062952)$ & $C_{2}$ & $(0.791616,0.019595,0.0062964)$ \\
& & $C_{3}$ & $(0.764811,0.017463,0.0062989)$ \\
\hline
\end{tabular}

and the eigenvalues $\left(\mathrm{Ex}^{*}, \mathrm{En}^{*}, \mathrm{He}^{*}\right)$ of the evaluation result in Table 10 are input into the forward cloud generator of MATLAB software, and a contrast cloud map of evaluation results and standard evaluation levels of the green protection effect of the subgrade slope in the DHV area of the SichuanTibet railway is obtained, as shown in Figure 4.

In Figure 4, the blue map is the cloud map of the standard evaluation level "excellent," the pink map is the cloud map of the standard evaluation level "good," the yellow map is the cloud map of the standard evaluation level "general," the green map is the cloud map of the standard evaluation level "poor," the red map is the cloud map of the standard evaluation level "very poor," and the black map is the cloud map of the evaluation result. The figure shows that the cloud map of the evaluation result is basically contained in the cloud map of the standard evaluation level "good," and the evaluation value corresponding to the vertex of the evaluation result cloud map (i.e., the expectation $\mathrm{Ex}^{*}=0.781816$ of the evaluation result) is close to the expectation $\mathrm{Ex}^{0}=0.7790$ of the standard evaluation level "good." Therefore, the green protection effect of this railway subgrade slope in the DHV area is "good." The evaluation result is consistent with the actual protection effect of the expert on-site investigation.

To further determine the accuracy of the evaluation results, an evaluation cloud map comparing the first-level indexes of the soil matrix quality $C_{1}$, vegetation community quality $C_{2}$, and protective performance of the slope $C_{3}$ with the standard evaluation level is also generated, as shown in Figures 5-7.

Figures 5-7 show that the evaluation cloud maps of the soil matrix quality $C_{1}$, vegetation community quality $C_{2}$, and protective performance of the slope $C_{3}$ (i.e., the first-level indexes) are all between the cloud maps of the standard evaluation levels "good" and "excellent" and very close to the cloud map of the standard evaluation level "good." In particular, the evaluation cloud maps of $C_{1}$ and $C_{3}$ are basically completely contained in the cloud map of the standard evaluation level "good," such that the evaluation level of the three first-level indexes is "good." By determining the evaluation level of the first-level indexes, it is further confirmed that the green protection effect of the railway subgrade slope in this section is "good."

\section{Discussion}

The evaluation index system basically contains all the factors that affect the green protection effect for the railway subgrade slope in the DHV area, and the evaluation results are thus convincing. However, the limitation of the evaluation index system is that half the evaluation indexes are qualitative and half are quantitative; i.e., there are too few

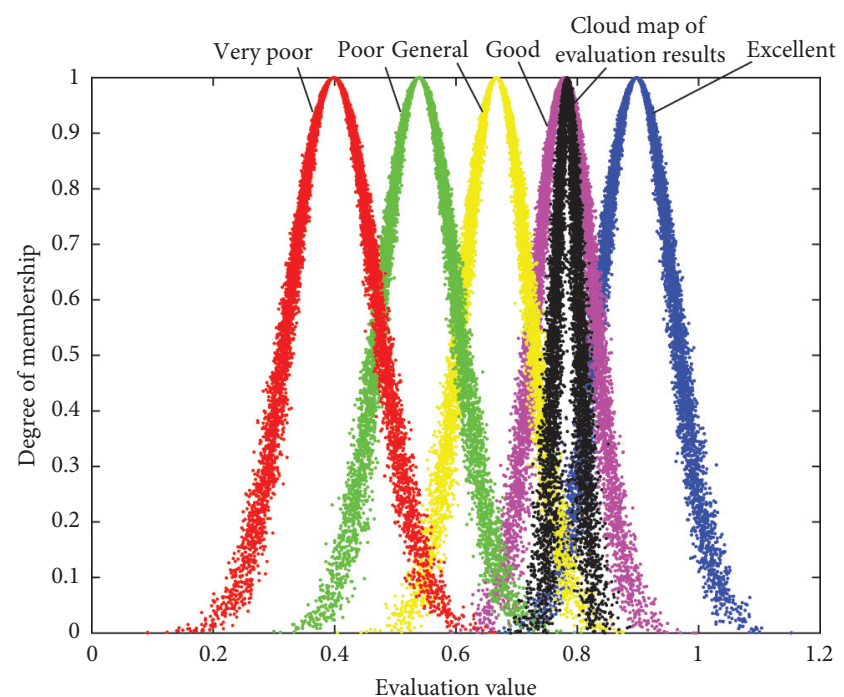

FIgURE 4: Contrast cloud map of evaluation results and standard evaluation levels.

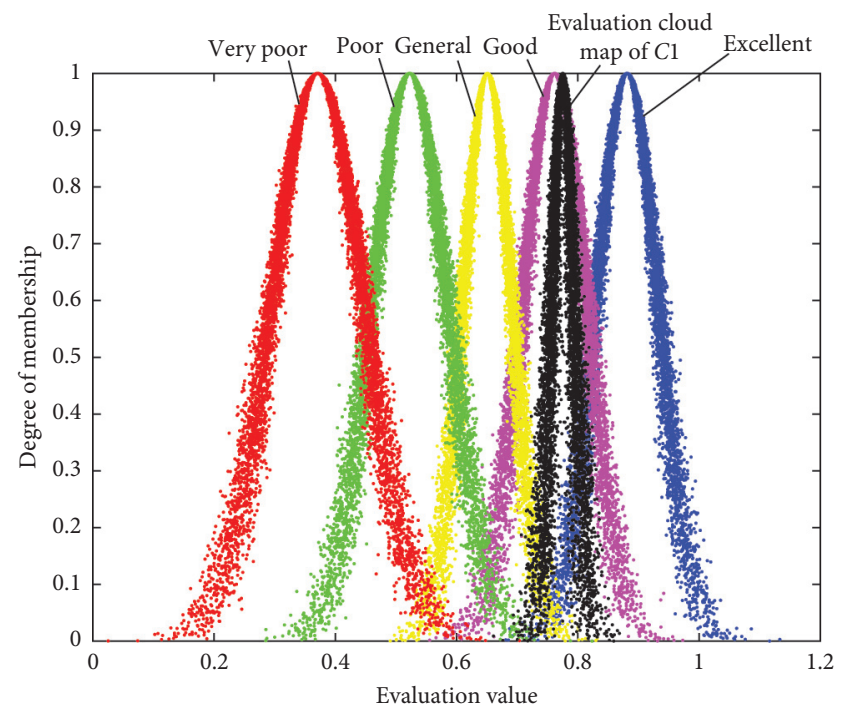

FIgURE 5: Contrast cloud map of the evaluation result of $C_{1}$ and standard evaluation level.

quantitative indexes and too many qualitative indexes. In addition, the qualitative indexes are only giving their status levels according to the actual conditions observed by experts, which makes the assessment of qualitative indexes too subjective because of the influence of experts themselves. Therefore, how to measure and evaluate qualitative indexes needs further research to improve the accuracy of evaluation 


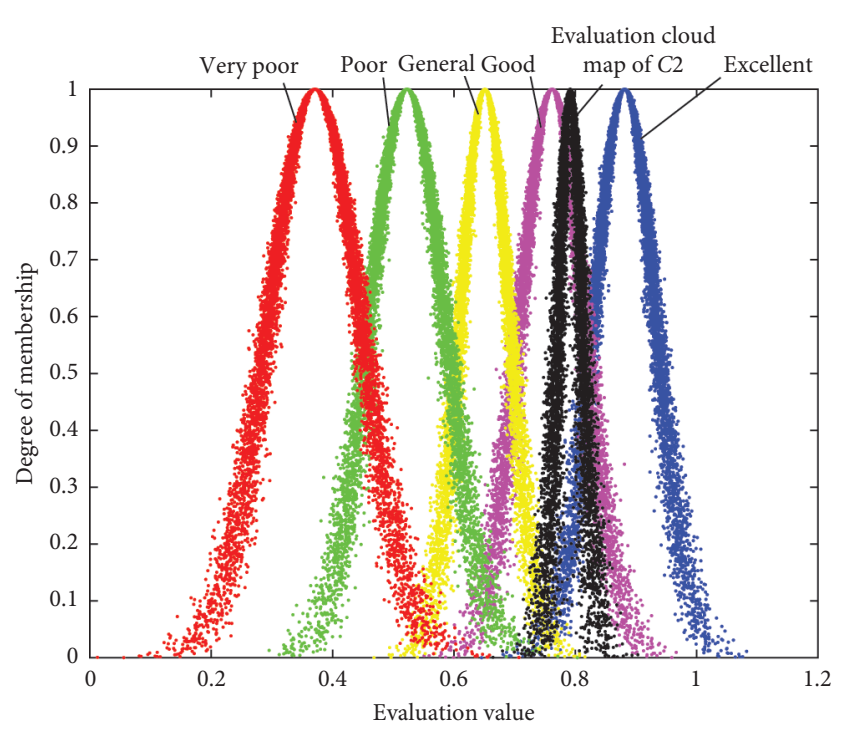

Figure 6: Contrast cloud map of the evaluation result of $C_{2}$ and standard evaluation level.

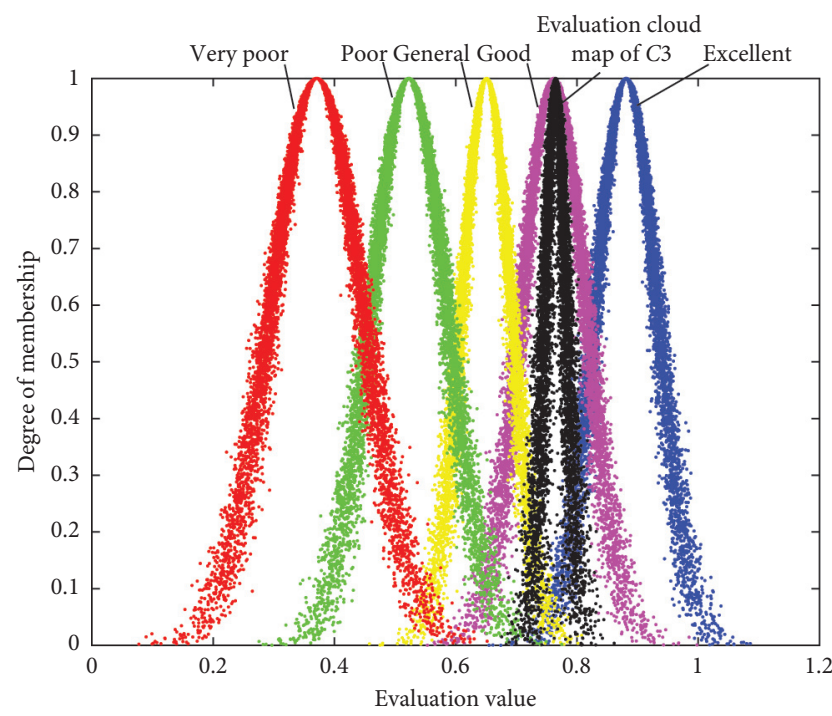

Figure 7: Contrast cloud map of the evaluation result of $C_{3}$ and standard evaluation level.

results. Moreover, the evaluation index system is established for DHV areas with fragile ecological environments, and some indexes used in the evaluation system may not be applicable to other regions. For instance, duration of soil PWP $C_{14}$, soil expansion and contraction $C_{18}$, ability of vegetation to resist drought and heat $C_{26}$, and slope resistance to leaching erosion $C_{31}$ indexes are unique to $\mathrm{DHV}$ areas. The DHV areas are mostly torrid red soil, and soil water content at a depth of $20 \mathrm{~cm}$ for 7 to 8 months of the year is in a PWP state, which causes plants to die for lack of water. Although index $C_{18}$ may be applicable to other areas, soil expansion and contraction is particularly prominent in DHV areas. There are distinct dry and wet seasons in the DHV area, which results in the large expansion and contraction of soil due to changes in dry and wet conditions, resulting in cracks in the soil. In severe cases, the plants will die due to root fracture. The DHV area is extremely dry and hot, and the slope protection vegetation must be able to resist drought and hot climate in order to play a better slope protection role; therefore, the index $C_{26}$ is exclusively suitable for the study area. Although the DHV areas are very dry, the rainfall is very concentrated during the wet season, which makes the water infiltrated into the soil too large, dissolving the nutrients from the soil; therefore, index $C_{31}$ is also unique to the areas. The four indexes above are selected based on the unique geological environment and climatic characteristics of the DHV areas, which is relatively applicable only to the DHV areas. The evaluation system therefore needs to be improved for the green protection effect of railway subgrade slopes in other areas according to the actual situation in those areas.

The advantage of the present study is that a subjective weighting method and objective weighting method are combined to determine the weighting, such that the weight distribution is based on not only the effects of subjective human factors but also the effects of objective sample data, which makes the weight more comprehensive and reasonable. In references [41, 44], only a subjective weight method (AHP) is used, which relies too much on a person's information and fails to consider the importance of experts themselves. However, this paper uses the IGAHP method not only to consider the importance of the experts themselves but also to use the GT to combine the vector-anglecosine method with the IGAHP method so that the weights are more accurate. As for the evaluation model, reference [41] uses the grey system theory to calculate the grey level of the evaluation object by constructing the definite weighted function so as to evaluate the quality of slope protection engineering. Reference [44] uses fuzzy comprehensive evaluation method to calculate the comprehensive membership of the research object and determine the slope protection effect of the slope protection project by comparison with the standard membership. Both methods above solve the problem by considering the uncertainty and ambiguity of the research problem, and they have their own advantages. However, the MCGC model not only considers the fuzziness of the research problem but also evaluates it by calculating the sample data and drawing the contrast cloud map with MATLAB software. Furthermore, the model intuitively reflects the evaluation results through a combination of graphics and text, making the evaluation process more detailed and the evaluation results more accurate.

The evaluation results are in good agreement with the actual situation of the railway subgrade slope protection project in the studied section of the Sichuan-Tibet railway. This shows that the construction of the subgrade slope protection project complies with the requirements of China to protect the ecological environment when constructing railways. However, only six experts were invited to collect data, and small data sample resulted in slight bias in the calculation results. Therefore, in future research, it will be necessary to take as many sample data as possible to improve the accuracy of the results. Moreover, it is worth mentioning 
that the present research provides a theoretical basis for government to evaluate the quality of the green protection engineering of the railway subgrade slope in the DHV area.

\section{Conclusion}

(1) Environmental and geographical characteristics were analyzed and combined with the characteristics and requirements of railway subgrade slope protection, and an evaluation index system for the green protection effect of railway subgrade slopes in a DHV area was then established.

(2) Subjective and objective weights of the evaluation indexes were calculated using the IGAHP method and the vector-angle-cosine method, respectively, and GT was then used to combine the subjective and objective weights such that the combined weight not only highlights the particularity of the DHV area but also eliminates the conflict and difference between subjective and objective factors. In addition, an evaluation model was established using the MCGC method, and an evaluation cloud map was drawn with MATLAB software to analyze the evaluation result.

(3) The GT combination weighting model and MCGC evaluation model were used to evaluate the protection effect in a practical engineering case, which is a section of subgrade slope protection engineering passing through the DHV area of the Sichuan-Tibet railway. The evaluation results are in good agreement with the actual engineering situation, demonstrating the applicability and effectiveness of the model.

\section{Data Availability}

The data used to support the findings of the study are available from the corresponding author upon request.

\section{Conflicts of Interest}

The authors declare that they have no conflicts of interest.

\section{Acknowledgments}

This research was supported by the National Natural Science Foundation of China (grant no. 51768034).

\section{References}

[1] K. Wu, Q. Sheng, and Y. H. Zhang, "Development of real-time remote monitoring and forecasting system for geological disasters at subgrade slopes of mountainous highways and its application," Rock and Soil Mechanics, vol. 31, no. 11, pp. 3683-3687, 2010.

[2] D. Wang and J. J. Wang, "Selection of optimal slope protection plants based on fuzzy neural network," Journal of Northeast Forestry University, vol. 7, pp. 116-119, 2011.

[3] K.-S. Cha and T.-H. Kim, "Evaluation of slope stability with topography and slope stability analysis method," KSCE Journal of Civil Engineering, vol. 15, no. 2, pp. 251-256, 2011.
[4] T. Yang, S. C. Li, and H. L. Sun, "Key issues in research of ecoengineering for rock slope protection," Research of Soil and Water Conservation, vol. 6, pp. 14-16, 2007.

[5] Y.-C. Hwang, "Change of slope stability due to slope inclination and surface conditions," Journal of the Korean Geotechnical Society, vol. 31, no. 2, pp. 5-11, 2015.

[6] H. G. Jeong and Y. S. Seo, "Slope stability analysis and landslide hazard assessment in tunnel portal area," Journal of Korean Tunnelling and Underground Space Association, vol. 15, no. 4, pp. 387-400, 2013.

[7] J. H. Shin, H. M. Kim, and K. J. Jang, "Effect of pore water pressure on slope stability by using coupled finite element analysis," Journal of the Korean Geotechnical Society, vol. 25, no. 2, pp. 25-35, 2009.

[8] Y. A. Makarova and A. Y. Manukovskiy, "The effect of floodwater on slope disturbance," Bulletin of Higher Educational Institutions. Lesnoi Zhurnal (Forestry Journal), vol. 2, no. 2, pp. 70-76, 2018.

[9] J. F. Lian, Q. Luo, and T. Xie, "Mechanics effects of frame protection for shallow stability of soil slope and frame structure optimization," Journal of the China Railway Society, vol. 39, no. 7, pp. 134-140, 2017.

[10] W. X. Cui, "Research on the optimization of high-speed railway earthwork slope protections," Journal of Railway Engineering Society, vol. 10, pp. 17-22, 2019.

[11] S. Cheng, "Application of assembled skeleton slope protection in subgrade slope protection of Qinghai-Tibet railway," Railway Engineering, vol. 10, pp. 69-71, 2003.

[12] A. Wang, "Application of stabilizing piles with pre-stressed anchor cables retaining structure in subgrade slope protection," Highway Engineering, vol. 43, no. 1, pp. 145-148, 2018.

[13] G. Y. Wang, Y. Wang, and N. Xu, "Test and study on erosion characteristics of 3D geonet protection slop," Research of Soil and Water Conservation, vol. 24, no. 1, pp. 79-83, 2017.

[14] Z.-X. Yan, Z.-H. Ren, C.-M. Yan, P. Jiang, and H.-Y. Wang, "Study on original ecological tridimensional slope vegetation," Journal of Mountain Science, vol. 10, no. 6, pp. 932-939, 2013.

[15] H. Y. Liu, L. Liu, and X. J. Li, "Comprehensive benefit evaluation on the protection technique of plant fiber blanket on the road side slope," Journal of Soil and Water Conservation, vol. 33, no. 1, pp. 345-352, 2019.

[16] Y. W. Luo, P. Han, and G. Y. Zheng, "Research on the environment-friendly railway route selection of Ya'an-kangding section of Sichuan-Tibet railway," Journal of Railway Engineering Society, vol. 8, pp. 1-5, 2017.

[17] S. Q. Luo, F. D. Xu, and S. F. Li, "Ecological restoration measures for difficult sites in dry and hot mountain valleys in southwest China," Journal of Green Science and Technology, vol. 14, pp. 11-13, 2019.

[18] Y. Dong, D. Xiong, Z. Su et al., "The influences of mass failure on the erosion and hydraulic processes of gully headcuts based on an in situ scouring experiment in dry-hot valley of China," Catena, vol. 176, pp. 14-25, 2019.

[19] A. L. Khan, G. R. McMeeking, J. P. Schwarz et al., "Nearsurface refractory black carbon observations in the atmosphere and snow in the McMurdo dry valleys, Antarctica, and potential impacts of foehn winds," Journal of Geophysical Research: Atmospheres, vol. 123, no. 5, pp. 2877-2887, 2018.

[20] C. R. Ou, Q. K. Zhu, and Y. Y. Sun, "Research progress in the landcape pattern of dry-hot valley in southwest China," Journal of West China Forestry Science, vol. 44, no. 6, pp. 137-142, 2015.

[21] L. Rong, X. Duan, G. Zhang, Z. Gu, and D. Feng, "Impacts of tillage practices on ephemeral gully erosion in a dry-hot valley 
region in southwestern China," Soil and Tillage Research, vol. 187, pp. 72-84, 2019.

[22] T. Hubble, S. Clarke, A. Stokes, and C. Phillips, "4th international conference on soil bio and eco-engineering (SBEE2016) 'the use of vegetation to improve slope stability'," Ecological Engineering, vol. 109, pp. 141-144, 2017.

[23] G. Tardío and S. B. Mickovski, "Implementation of eco-engineering design into existing slope stability design practices," Ecological Engineering, vol. 92, pp. 138-147, 2016.

[24] J. Geris, D. Tetzlaff, J. McDonnell, and C. Soulsby, "The relative role of soil type and tree cover on water storage and transmission in northern headwater catchments," Hydrological Processes, vol. 29, no. 7, pp. 1844-1860, 2015.

[25] D.-H. Xiong, H.-Y. Zhou, Z. Yang, and X.-B. Zhang, "Slope lithologic property, soil moisture condition and revegetation in dry-hot valley of Jinsha river," Chinese Geographical Science, vol. 15, no. 2, pp. 186-192, 2005.

[26] Q. B. Chen, K. Q. Wang, and Y. M. Li, "Effect of different vegetation types on soil amelioration in dry-hot valley of Jinshajiang River basin," Journal of Soil Water Conservation, vol. 19, no. 2, pp. 67-70, 2003.

[27] A. Ghosh, A. Singh, R. Kumar et al., "Soil enzymes and microbial elemental stoichiometry as bio-indicators of soil quality in diverse cropping systems and nutrient management practices of Indian Vertisols," Applied Soil Ecology, vol. 145, 2020.

[28] Z. Q. Liu, N. J. Lang, and K. Q. Wang, "Research on soil infiltration law of different landues types in Jinsha River dryhot valley," Advanced Materials Research, vol. 726-731, pp. 3867-3871, 2013.

[29] N. Y. Musadji, L. Lemee, L. Caner, G. Porel, P. Poinot, and C. Geffroy-Rodier, "Spectral characteristics of soil dissolved organic matter: long-term effects of exogenous organic matter on soil organic matter and spatial-temporal changes," Chemosphere, vol. 240, pp. 124808-124814, 2020.

[30] L. Xiao, P. Li, P. Shi, and Y. Liu, "Soil nutrient stoichiometries and enzymatic activities along an elevational gradient in the dry-hot valley region of southwestern China," Archives of Agronomy and Soil Science, vol. 65, no. 3, pp. 322-333, 2018.

[31] X.-w. Duan, X. Han, J.-m. Hu, D.-t. Feng, and L. Rong, “A novel model to assess soil productivity in the dry-hot valleys of China," Journal of Mountain Science, vol. 14, no. 4, pp. 705-715, 2017.

[32] N. F. Zhao, W. M. Ye, B. Chen, Y. G. Chen, and Y. J. Cui, "Modeling of the swelling-shrinkage behavior of expansive clays during wetting-drying cycles," Acta Geotechnica, vol. 14, no. 5, pp. 1325-1335, 2019.

[33] Z. Xiang, "Degradation of ecosystem and ways of its rehabilitation and reconstruction in dry-hot valley," Resources and Environment in the Yangtze Basin, vol. 9, no. 3, pp. 376-383, 2000.

[34] J. Pei, W. Yang, and Y. P. Cai, "Relationship between vegetation and environment in an arid-hot valley in southwestern China," Sustainability, vol. 10, no. 12, pp. 1-14, 2018.

[35] H. Wu, D.-H. Xiong, L. Xiao et al., "Effects of vegetation coverage and seasonal change on soil microbial biomass and community structure in the dry-hot valley region," Journal of Mountain Science, vol. 15, no. 7, pp. 1546-1558, 2018.

[36] Y. F. Dong, D. H. Xiong, Z. G. Su et al., "The distribution of and factors influencing the vegetation in a gully in the dry-hot Valley of southwest China," Catena, vol. 116, pp. 60-67, 2014.

[37] S. Peng, A. Chen, H. Fang, J. Wu, and G. Liu, "Effects of vegetation restoration types on soil quality in Yuanmou dry-hot valley, China," Soil Science and Plant Nutrition, vol. 59, no. 3, pp. 347-360, 2013.

[38] Y. Cui, J. Li, A. Chen et al., "Fractal dimensions of trapped sediment particle size distribution can reveal sediment retention ability of common plants in a dry-hot valley," Catena, vol. 180, pp. 252-262, 2019.

[39] X. Hu, Z. Chen, and C. J. Li, "Grade evaluation on quality of slope protection engineering by vegetation," Bulletin of Soil and Water Conservation, vol. 33, no. 3, pp. 180-185, 2013.

[40] The First Editing Room of Standards Press of China, Compilation of National Standards for Soil and Water Conservation, China Standards Press, Beijing, China, 2011.

[41] B. T. Wan, X. Y. Bao, and A. C. Li, "Quality evaluation system and application of ecological slope protection project based on unascertained-set pair coupling," Bulletin of Soil and Water Conservation, vol. 39, no. 2, pp. 108-114, 2019.

[42] F. Long, S. C. Li, and H. L. Sun, "Evaluation indicator system for effect of eco-engineering for rock slope protection and its application," Chinese Journal of Rock Mechanics and Engineering, vol. 28, no. supp.1, pp. 3095-3101, 2009.

[43] J. An, T. X. Xu, and X. Zeng, "Equipment quality condition assessment under fusion information based on combination weighting," Control and Decision, vol. 33, no. 9, pp. 16931698, 2018.

[44] S. Chakraborty and S. Mukhopadhyay, "Assessing flood risk using analytical hierarchy process (AHP) and geographical information system (GIS): application in Coochbehar district of West Bengal, India," Natural Hazards, vol. 99, no. 1, pp. 247-274, 2019.

[45] H. Shen, J. C. Xie, and J. X. Li, "Combined hydrological forecasting method based on vector cosine," System Engineering Theory and Practice, vol. 32, no. 7, pp. 1591-1597, 2012.

[46] S. Abapour, B. Mohammadi-Ivatloo, and M. T. Hagh, "Robust bidding strategy for demand response aggregators in electricity market based on game theory," Journal of Cleaner Production, vol. 243, p. 118393, 2020.

[47] K. X. Huang, C. J. Zhou, Y. Q. Qin, and W. Tu, "A gametheoretic approach to cross-layer security decision-making in industrial cyber-physical systems," IEEE Transactions on Industrial Electronics, vol. 67, no. 3, pp. 2371-2379, 2020.

[48] Z. L. Wei, C. L. Chen, and Y. Q. Dai, "Research on quality evaluation of scheme for equipment system based on IGAHPIEW-GT-MCGC," Fire Control \& Command Control, vol. 42, no. 11, pp. 114-120, 2017.

[49] Z. Sun and L. H. Zhao, "Reliability real-time evaluation of JTC based on cloud theory," Journal of the China Railway Society, vol. 41, no. 2, pp. 89-96, 2019.

[50] Z. Huang, H. L. Fu, and J. B. Zhang, "Comprehensive evaluation model of shield tunnel construction risk based on cloud theory," Journal of Railway Science and Engineering, vol. 15, no. 11, pp. 3012-3020, 2018.

[51] L. P. Liang, Y. G. Liu, and Z. H. Tang, "Geologic hazards susceptibility assessment based on weighted information value a case study in Ludi county, Sichuan province," Bulletin of Soil and Water Conservation, vol. 39, no. 6, pp. 176-182, 2019. 